\title{
Ovarian Cancer and Primary Peritoneal Carcinoma cM0 TNM Finding v7
}

National Cancer Institute

\section{Source}

National Cancer Institute. Ovarian Cancer and Primary Peritoneal Carcinoma CMO TNM

Finding V7. NCI Thesaurus. Code C89662.

Ovarian cancer or primary peritoneal carcinoma without evidence of distant metastasis.

(from AJCC 7th Ed.) 